Clara Muniz da Silva de Almeida

\title{
Análise por XPS e AFM da superfície de blendas de PVC/PHB
}

DISSERTAÇÃO DE MESTRADO

DEPARTAMENTO DE FÍSICA

Programa de Pós-Graduação em Física 


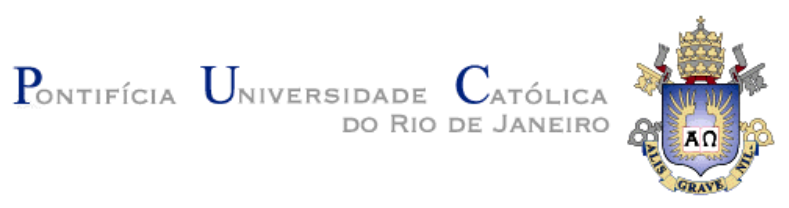

Clara Muniz da Silva de Almeida

\section{Análise por XPS e AFM da superfície de blendas de PVC/PHB.}

Dissertação de Mestrado

Dissertação apresentada ao Programa de Pós-graduação em Física da PUC-Rio como requisito parcial para obtenção do título de Mestre em Física.

Orientador: Prof. Rodrigo Prioli Menezes 


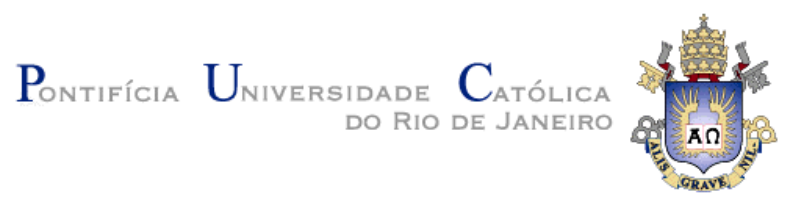

Clara Muniz da Silva de Almeida

\title{
Análise por XPS e AFM da superfície de blendas de PVC/PHB
}

Dissertação apresentada como requisito parcial para obtenção do grau de Mestre pelo Programa de Pós-Graduação em Física do Departamento de Física do Centro Técnico Científico da PUC-Rio. Aprovada pela Comissão Examinadora abaixo assinada.

\author{
Prof. Rodrigo Prioli Menezes \\ Orientador \\ Departamento de Física - PUC-Rio
}

Prof. Rubén Jesus Sánchez Rodriguez

Co-Orientador Laboratório de Materiais Avançados - UENF

Prof. Gilson Brand Baptista

Prof. Suzana Isabel Zanette de Caride

CBPF

Prof. José Eugenio Leal

Coordenador Setorial do CTC - PUC-Rio.

Rio de Janeiro, 29 de Março de 2005. 
Todos os direitos reservados. É proibida a reprodução total ou parcial do trabalho sem autorização da universidade, do autor e do orientador.

\section{Clara Muniz da Silva de Almeida}

Graduou-se em Física na Pontifícia Universidade Católica do Rio de Janeiro - PUC-Rio em 2002. Atualmente é aluna de doutorado na PUC-Rio.

Ficha catalográfica

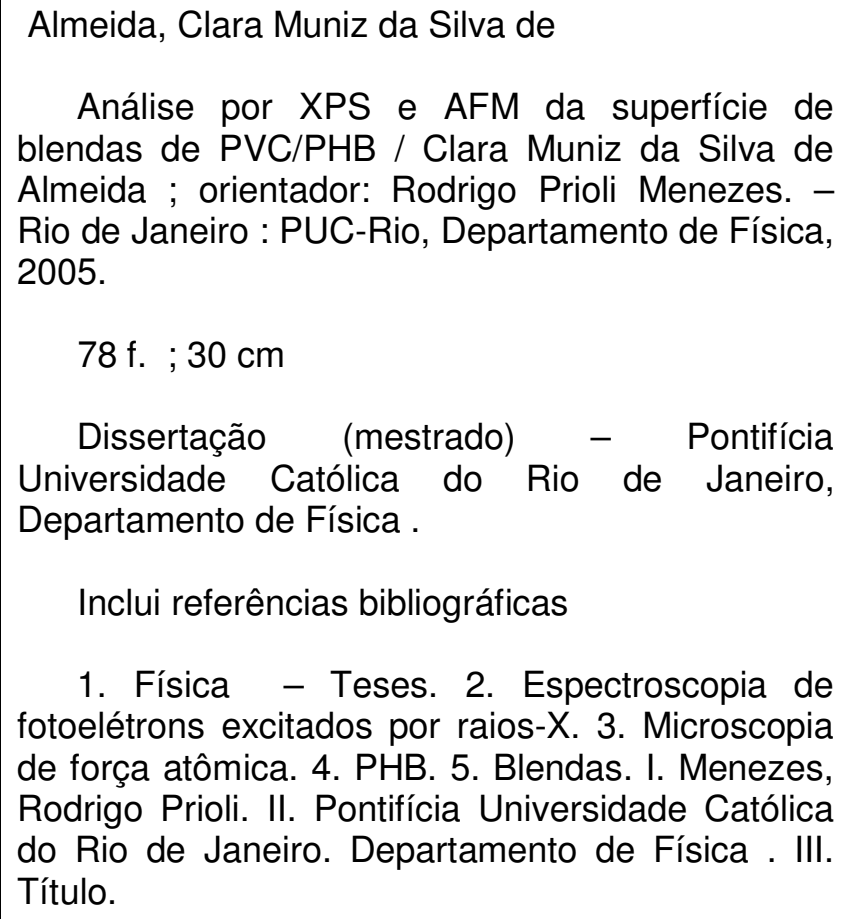
blendas de PVC/PHB / Clara Muniz da Silva de Almeida ; orientador: Rodrigo Prioli Menezes. Rio de Janeiro : PUC-Rio, Departamento de Física, 2005.

$78 \mathrm{f.} ; 30 \mathrm{~cm}$

Dissertação (mestrado) - Pontifícia Universidade Católica do Rio de Janeiro, Departamento de Física .

Inclui referências bibliográficas

1. Física - Teses. 2. Espectroscopia de fotoelétrons excitados por raios-X. 3. Microscopia de força atômica. 4. PHB. 5. Blendas. I. Menezes, Rodrigo Prioli. II. Pontifícia Universidade Católica do Rio de Janeiro. Departamento de Física . III. Título.

CDD: 530 
Aos meus avós, tios, primos, pai e irmãozinho. Especialmente à minha mãe, meu exemplo. 


\section{Agradecimentos}

Ao Professor Rodrigo Prioli Menezes, que me acolheu repentinamente, acreditou na minha capacidade e no meu trabalho. Pela amizade criada e por ter me apresentado novas possibilidades. Pelos conhecimentos compartilhados, pelo esforço conjunto, pelas críticas e pela atenção.

Ao Professor Gilson Brand Baptista, muito mais que um orientador. Por acreditar em mim e me incentivar sempre. Pelos conselhos, pelos ensinamentos e pela amizade.

Ao Prof. Ruben Sanchez e ao Marcelo O. Siqueira pela ajuda e pelo auxílio e pelo tempo gasto comigo.

Ao Henrique e ao Robert, pelas inúmeras risadas e por estarem sempre por perto para minhas dúvidas. Pela amizade.

Agradeço aos meus amigos que me acompanham nessa jornada e que sem eles por perto, não sei se seria possível chegar aqui: Flávia, Samantha, Pedro, Lucas,... e aos engenheiros, enfim todos que estiveram e estão por perto.

A todos os professores e funcionários do Departamento de Física que contribuíram no meu crescimento acadêmico.

Ao CNPq e a PUC-Rio pelos auxílios concedidos, sem os quais este trabalho não teria sido realizado. 


\section{Resumo}

Almeida, C. M. Análise por XPS e AFM da superfície de blendas de PVC/PHB. Rio de Janeiro, 2005. 78p. Dissertação de Mestrado - Departamento de Física, Pontifícia Universidade Católica do Rio de Janeiro.

Neste trabalho, foram estudadas blendas formadas pelo polímero Policloreto de Vinila (PVC) e pelo polímero biodegradável Poli Hidroxibutirato (PHB), com diferentes concentrações relativas de cada polímero. As blendas foram preparadas em forma de membrana, com concentrações de PHB variando de 2 a 40\%. A técnica utilizada para o preparo das amostras foi a evaporação do solvente, sendo dicloroetano o solvente utilizado. A composição química das superfícies das blendas foi caracterizada utilizando a técnica de espectroscopia de fotoelétrons induzida por raios-X (XPS). A morfologia e as propriedades mecânicas das amostras foram estudadas com o auxílio da microscopia de força atômica (AFM). Medidas de calorimetria diferencial exploratória foram realizadas a fim de conhecermos a miscibilidade das blendas formadas. Nas blendas formadas observou-se uma segregação de PHB para a superfície, sendo o percentual de PHB na superfície na faixa de $18 \pm 2 \%$, mesmo para blendas com mais baixa concentração relativa de PHB. Entretanto o grau de cristalização do PHB em cada blenda aumenta com a incorporação do polímero na blenda. Foi observado um aumento na dureza conforme aumentamos a concentração de PHB na blenda. As blendas foram caracterizadas como parcialmente miscíveis.

\section{Palavras - chave}

Física - Dissertação; Espectroscopia de fotoelétrons excitados por raios-X; microscopia de força atômica; PHB; blendas. 


\section{Abstract}

Almeida, C. M. XPS and AFM analysis of PVC/PHB blends surfaces. Rio de Janeiro, 2005. 78p. Msc. Dissertation - Departamento de Física, Pontifícia Universidade Católica do Rio de Janeiro.

In this work, the study of polymer blends surfaces formed by a biodegradable polymer (poly3-hydroxybutyrate, PHB) and PVC (polyvinyl chloride) with different molar percentages of PHB is presented. The blends samples were prepared as membranes with PHB molar concentrations from 2 to $40 \%$. The technique used to prepare the membranes was the solvent-casting technique, using dichloroethene as solvent. The chemical composition of the blends samples surfaces were characterized by X-ray photoelectron spectroscopy (XPS). Their morphologies and mechanical properties were studied by atomic force microscopy (AFM). Differential scanning calorimeter measures were obtained in order to know the blends miscibility. A PHB surface segregation of $18 \pm 2 \%$ was observed, even for the blends with lower PHB concentrations. However, the degree of the PHB crystallization in each blend increases with the increasing incorporation of PHB in the blend volume. An increase in the blends hardness was observed while the PHB blends concentration increases. The blends were founded to be partially miscible.

\section{Keys - Words}

Physics - Dissertation; X-ray photoelectron spectroscopy; Atomic Force Microscopy; PHB; blends. 


\section{Sumário}

1. Introdução 16

2. Procedimentos experimentais 23

2.1. Preparação das blendas 23

2.2. Espectroscopia de elétrons induzida por raios-X (XPS) 25

2.2.1. Princípios básicos 25

2.2.2. Instrumentação utilizada 26

2.2.3. Calibração do equipamento utilizado nas análises de XPS 29

2.2.4. Análise semi-quantitativa através da técnica de XPS 30

2.3. Ângulo de Contato 31

2.3.1. Princípios básicos 31

2.3.2. Instrumentação utilizada 32

2.4. Microscopia atômica de força (AFM) 34

2.4.1. Princípios básicos 34

2.4.2. Instrumentação utilizada 37

2.5. Calorimetria diferencial exploratória 42

2.5.1. Princípios básicos 42

2.5.2. Instrumentação utilizada 43

3. Resultados obtidos 45

3.1. Espectroscopia de elétrons induzida por raios-X (XPS) 45

3.1.1. Análise semi-quantitativa através da técnica de XPS 50

3.2. Ângulo de Contato 51

3.2.1. Ângulo de contato estático (SCA) 51

3.2.2. Ângulo de contato dinâmico (DCA) 53

3.3. Microscopia de Força Atômica (AFM) 56

3.3.1. Morfologia 56

3.3.2. Medindo qualitativamente as propriedades 
mecânicas das blendas com o AFM

65

3.4 Calorimetria Diferencial Exploratória

69

4. Conclusão e perspectivas futuras

72

Referências

75

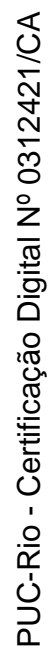




\section{Lista de figuras}

Figura 1.1 - Fórmula estrutural dos PHAs 17

$\begin{array}{ll}\text { Figura } 1.2 \text { - Fórmula estrutural do PHB } & 17\end{array}$

Figura 2.1 - Foto tirada do interior da câmara de XPS utilizada 27

Figura 2.2 - Foto do porta amostras de prata utilizado para prender a membrana polimérica para fazer as análises de XPS 28

Figura 2.3 - Curva de calibração do analisador de elétrons utilizado para XPS

Figura 2.4 - Esquema representativo das tensões superficiais e do ângulo de contato entre uma gota e uma superfície

Figura 2.5 - Aparato experimental utilizado para a medida de ângulo de contato

Figura 2.6 - Gráfico da força resultante em função da distância ponta-superfície

Figura 2.7 - Microscópio de força atômica utilizado

Figura 2.8 - Indentações realizadas com correções de diferentes ângulos a fim de identificar a melhor correção

Figura 2.9 - Perfis topográficos das indentações realizadas com correção no ângulo de torção igual a 30ํ⒜, 20ํ⒝ e $10^{\circ}(\mathrm{c})$

Figura 2.10 - Exemplo do processo de equalização realizado nas indentações para realçar a diferença de alturas da imagem. O intervalo de alturas selecionado neste histograma foi evidenciado com as setas tracejadas

Figura 2.11 - Exemplo do procedimento utilizado para o cálculo da área das indentações realizadas

Figura 2.12 - Esquema representando a célula utilizada nas medidas de DSC

Figura 2.13 - Suporte utilizado para as medidas de DSC 
Figura 2.14 - Equipamento de DSC utilizado

Figura 3.1 - Espectros de alta resolução na faixa de energia dos fotoelétrons do carbono (a) para o PVC, e os espectros da região de energias dos fotoelétrons do carbono (b) e do oxigênio (c) para o PHB

Figura 3.2 - Espectros de alta resolução na faixa de energia dos fotoelétrons do carbono para as blendas 2,4,5,8,e 12\% em a-e respectivamente, e os espectros da região de energias do oxigênio para as mesmas blendas $f-j$

Figura 3.3 - Fotos das gotas utilizadas para a medida do ângulo de contato para o $\mathrm{PVC}(\mathrm{a})$, blenda $05 \%$ (b) e $40 \%(\mathrm{c})$, e para o PHB puro(d)

Figura 3.4 - Variação do ângulo de contato em função do tempo para os polímeros puros $\mathrm{PHB}(\mathrm{a})$ e $\mathrm{PVC}(\mathrm{b})$, e para as blendas 2(c), 5(d), 12(e) e 40\%(f). Para as blendas foi feito um ajuste linear, e para os polímeros puros fizemos um ajuste exponencial

Figura 3.5 - Variação do ângulo de contato em função da concentração de PHB nas blendas. Cada curva representa um tempo diferente de observação da gota sobre a superfície 55

Figura 3.6 - Esquema da estrutura cristalina do PHB no plano AC. 57

Figura 3.7 - Imagem obtida pelo AFM da blenda 10\%. Escala em z variando de $0-214 \mathrm{~nm}$.

Figura 3.8 - Imagem de microscopia ótica da blenda 5\%, onde é possível observar os esferulitos espalhados por toda a superfície

Figura 3.9 - Imagens $100 \mu \mathrm{m}^{2}$ da topografia do (a) PVC e do (b) PHB. Escala em z variando de $0-15,5 \mathrm{~nm}$ (a) e $0-558,8 \mathrm{~nm}$ (b). $\quad 59$

Figura 3.10 - Imagens $250 \mu \mathrm{m}^{2}$ (a) e $100 \mu \mathrm{m}^{2}$ (b) da topografia da

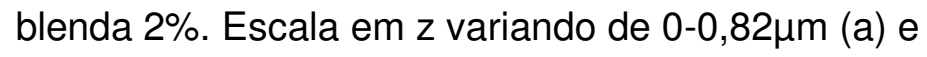


$0-320,3 n m(b)$

Figura 3.11 - Imagens $250 \mu \mathrm{m}^{2}$ (a) e $100 \mu \mathrm{m}^{2}$ (b) da topografia da

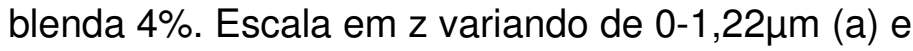
0-274,5nm (b)

60

Figura 3.12 - Imagens $250 \mu \mathrm{m}^{2}$ (a) e $100 \mu \mathrm{m}^{2}$ (b) da topografia da blenda $5 \%$. Escala em z variando de $0-638,6 \mathrm{~nm}$ (a) e $0-242 n m(b)$

Figura 3.13 - Imagens $250 \mu \mathrm{m}^{2}$ da topografia da blenda $6 \%$ (a) e da blenda $8 \%(b)$. Escala em z variando de $0-1,27 \mu \mathrm{m}$ (a) e 0-820nm (b).

Figura 3.14 - Imagens de topografia(a) e de força lateral(b) da blenda PVC/PHB5\%

Figura 3.15 - Ampliação das imagens de topografia (a) e de força lateral (b) da blenda $5 \%$, com seus respectivos perfis (c) da topografia e (d) da imagem de força lateral

Figura 3.16 - Fotos das gotas depositadas sobre as superfícies do PVC (a) e do PHB (b)

Figura 3.17 - Indentações realizadas na blenda PVC/PHB5\% 65

Figura 3.18 - Área das indentações realizadas em função da concentração de PHB na blenda

Figura 3.19 - Exemplo de perfil de profundidade de uma das indentações realizadas

Figura 3.20 - Indentações realizadas na blenda 10\% nas partes amorfa, cristalina e intermediária aparentes na superfície

Figura 3.21 - Resultado de DSC para as blendas 2, 4, 6, 8 e 12\% e para o PVC 


\section{Lista de tabelas}

Tabela 1.1 - Algumas propriedades físico-químicas do PHB, PHB-HV, PP e PVC

Tabela 2.1 - Relação das blendas estudadas

Tabela 3.1 - Tabela com o número de varreduras de cada espectros de alta resolução apresentado anteriormente

Tabela 3.2 - Parâmetros utilizados para o ajuste das curvas dos espectros de alta resolução da região do carbono para o PHB e PVC puros

Tabela 3.3 - Resultados das análises de XPS para a concentração de PHB na superfície da blenda em função da concentração de PHB na blenda

Tabela 3.4 - Tabela com os valores do ângulo de contato das blendas e dos polímeros puros

Tabela 3.5 - Coeficientes angulares das curvas do ângulo de contato em função do tempo para as blendas e para os polímeros puros

Tabela 3.6 - Área das indentações normalizadas a partir do maior valor obtido 


\title{
Lista de abreviações
}

\author{
AFM - Microscopia de Força Atômica \\ CAB - Cellulose Acetate Butyrate \\ CCD - "Charge Coupling Device" \\ $C_{P H B}$ - razão estequiométrica de carbono no monômero de PHB \\ $C_{P V C}$ - razão estequiométrica de carbono no monômero de PVC \\ DCA - Ângulo de contato dinâmico \\ DSC - Calorimetria Diferencial Exploratória \\ EPR - "ethylene-propylene rubber" \\ F - força normal entre ponta e superfície \\ FWHM - largura à meia altura \\ $\mathrm{K}$ - constante de mola do cantilever \\ K - coeficiente linear da reta ajustada \\ $O_{P H B}$ - razão estequiométrica de oxigênio no monômero de PHB \\ PBA - "poly(1,4-butylene adipate)" \\ PCHMA - "poly(cyclohexil methacrylate)" \\ PECH - "Poly(epichlorohydrin)" \\ PET - Polietileno Tereftalato \\ PHA - Polihidroxialcanoato \\ PHB - Polihidroxibutirato \\ PHB-HV - Polihidroxibutirato-co-hidroxivalerato \\ PHPV - "Poly(-3-hydroxy-5-phenylvalerate)" \\ PHU - "Polyhydroxyundecenoate" \\ PMMA - "Poly(methyl methacrylate)" \\ PP - Polipropileno \\ PVA - "Poly(vinyl alcohol)" \\ PVAc - Polivinil Acetato \\ PVB-VA - "Poly(vinyl butiral)-vinyl alcohol" \\ PVC - Policloreto de Vinila
}


PVDF - "Poly(vinylidene fluoride)"

$\mathrm{S}_{\mathrm{C}}$ - fator de sensibilidade do carbono

SCA - Ângulo de contato estático

$S_{O}$ - fator de sensibilidade do oxigênio

$T_{g}$ - Temperatura de transição vítrea

$T_{m}$ - Temperatura de fusão

$X$ - concentração relativa de PHB na superfície das blendas

XPS - Espectroscopia de Elétrons Induzida por Raios-X

$Y_{\mathrm{IV}}$ - tensão líquido-vapor

$Y_{\mathrm{sl}}$ - tensão sólido-líquido

$\mathrm{Y}_{\mathrm{sv}}$ - tensão sólido-vapor

$\Delta \mathrm{z}$ - deslocamento vertical do cantilever

$\theta$ - ângulo de contato 